Article

\title{
Analysis on Controllability Results for Wellposedness of Impulsive Functional Abstract Second-Order Differential Equation with State-Dependent Delay
}

\author{
Kulandhivel Karthikeyan ${ }^{1}$ (D), Dhatchinamoorthy Tamizharasan ${ }^{2} \mathbb{D}$ and Dimplekumar N. Chalishajar ${ }^{3, *}$ \\ 1 Department of Mathematics, Centre for Research and Development, KPR Institute of Engineering \\ and Technology, Tamilnadu, Coimbatore 641048, India; karthi_phd2010@yahoo.co.in \\ 2 Department of Mathematics, K. S. Rangasamy College of Technology, Tiruchengode, \\ Tamilnadu, Namakkal 641048, India; tamizhllim83@gmail.com \\ 3 Department of Applied Mathematics, Virginia Military Institute (VMI), 435, Mallory Hall, \\ Lexington, VA 24450, USA \\ * Correspondence: dipu17370@yahoo.com or chalishajardn@vmi.edu
}

check for updates

Citation: Karthikeyan, K.;

Tamizharasan, D.; Chalishajar, D.N. Analysis on Controllability Results for Wellposedness of Impulsive

Functional Abstract Second-Order Differential Equation with

State-Dependent Delay. Axioms 2021, 10, 188. https://doi.org/10.3390/

axioms 10030188

Academic Editor: Chris Goodrich

Received: 14 July 2021

Accepted: 11 August 2021

Published: 16 August 2021

Publisher's Note: MDPI stays neutral with regard to jurisdictional claims in published maps and institutional affiliations.

Copyright: (C) 2021 by the authors. Licensee MDPI, Basel, Switzerland. This article is an open access article distributed under the terms and conditions of the Creative Commons Attribution (CC BY) license (https:/ / creativecommons.org/licenses/by/ $4.0 /)$.

\begin{abstract}
The functional abstract second order impulsive differential equation with state dependent delay is studied in this paper. First, we consider a second order system and use a control to determine the controllability result. Then, using Sadovskii's fixed point theorem, we get sufficient conditions for the controllability of the proposed system in a Banach space. The major goal of this study is to demonstrate the controllability of an abstract second-order impulsive differential system with a state dependent delay mechanism. The wellposed condition is then defined. Next, we studied whether the defined problem is wellposed. Finally, we apply our results to examine the controllability of the second order state dependent delay impulsive equation.
\end{abstract}

Keywords: impulsive condition; cosine families; state dependent delay; wellposedness; controllability condition

MSC: Primary 34A08; 34B15; 93B05; Secondary 47H10

\section{Introduction}

The investigation of this paper guarantees that the defined problem is controllable on any interval. Such problems come from some physical applications as a natural generalization of the classical initial value problems. Consider the Cauchy problem with state-dependent delay and control:

$$
\begin{aligned}
y^{\prime \prime}(t) & =\mathscr{A} y(t)+\mathscr{F}\left(t, y_{\sigma_{1}\left(t, y_{t}\right)}, y_{\sigma_{2}\left(t, y_{t}\right)}^{\prime}\right)+B u(t), \quad t \in[0, b], \\
y_{0} & =\varphi \in \mathscr{B}=C([-\gamma, 0] ; X), y^{\prime}\left(0^{+}\right)=\varphi^{\prime}(0) \in X \\
\Delta y\left(t_{k}\right) & =I_{k}\left(x_{t_{k}}\right), \quad k=1,2, \ldots, n \\
\Delta y^{\prime}\left(t_{k}\right) & =J_{k}\left(x_{t_{k}}\right), \quad k=1,2, \ldots, n
\end{aligned}
$$

Here $(X,\|\cdot\|)$ is a Banach space, $\mathscr{A}$ denotes the infinitesimal generator of a strongly continuous cosine function of bounded linear operators $(C(t))_{t \in \mathbb{R}}$ on $(X,\|\cdot\|)$ and $\mathscr{F}(\cdot), \sigma_{j}(\cdot), j=1,2$ are appropriate functions; the function $y_{t}:(-\infty, 0] \rightarrow X, y_{t}(\theta)=y(t+$ $\theta)$, is a member of particular abstract phase space $\mathscr{B}$ defined later; $0<t_{1}<\cdots<t_{n}<b$ are annexed numbers; $\sigma_{j}: I \times \mathscr{B} \rightarrow(-\infty, b], j=1,2, I_{k}(\cdot), J_{k}(\cdot): \mathscr{B} \rightarrow X$ are suitable functions and $\Delta \xi(t)$ symbolizes the bounds of the function $\xi(\cdot)$ at $t$, and it is described as $\Delta \xi(t)=\xi\left(t^{+}\right)-\xi\left(t^{-}\right)$. 
The theory of impulsive differential equations has become a quite active area of research in the last few years. We refer to [1-3] for a preamble to this theory, which also comprises a varities of exciting examples and applications. Recently, the differential equation of second order with impulse systems on compact periods has been explored under inhomogeneous conditions by many authors, see [4,5]. From the publications [6,7], abstract partial differential equations with impulsive circumstances were investigated.

The concept of controllability is important in the research and application of control theory. Controllability has been investigated both with and without an impulse condition by many authors [8-11]. One of the most difficult aspects of controllability is demonstrating the presence of a control function, refer [4] and the existence of a controllable differential equation solution that steers the system's solution from its initial state to its final state; it varies over the entire space. Although, the controllability of second-order differential system with impulses has not nevertheless been adequately investigated thoroughly when the comparison of ordinary differential equations.

The impulsive differential system with state-dependent delay is a significant topic of current research. The state-dependent delay conditions in the differential equations with first order related collection of articles are wide-ranging. The references related to the case of ordinary differential equations described in finite-dimensional spaces given by Aiello, Freedman \& Wu [12] and the studies of the papers of [13-16] are also reviewed. Abstract differential equations and partial differential equations of the first order were investigated for the state-dependent delay condition in the papers provided by Hernandez, and et al. [17] and the research articles of [18-21]. The existence of solutions to the impulsive differential equation of second order with state-dependent delay has recently been investigated, refered to [22,23]. Also, the state dependent delay term for the parabolic and other ODE systems contributed by Krisztin et al. [24] and the cited papers [25-27] have been studied for the current research.

Buger and Martin [28] and Si's [29] papers on ordinary differential equations of second order with state-dependent delay are interesting. Abstract differential equations and partial differential equations of second order were used in the research of state-dependent delay, as evidenced by a review of a few papers, see [5,30-34]. Many of these articles use fixed point theorems to impart at least one mild solution using the condensing maps property, but the existence of exactly one solution to the given problem is not addressed, but except the paper cited [31]. For more about recent development of the related field, one can refer to $[10,11,35,36]$. The details of the function $y \rightarrow \mathscr{F}\left(\cdot, y_{\sigma_{j}\left(\cdot, y_{(\cdot)}\right)}\right), j=1,2$ are not Lipschitz in the space of continuous functions are collected from $[24,25,27]$. The goal of this article is to study the existence of controllability of the problem (1)-(4). Also, we show the wellposedness of the problem (1)-(4).

In some mathematical models of real-world processes, impulsive functional second order differential equations with state-dependent delays are becoming more important. Our research differs significantly from those of equations with a constant or time-dependent delay. We can represent numerous evolutionary processes using impulsive functional abstract second order differential equations with state-dependent delay in many disciplines of science, which is why the study of these equations has received a lot of interest in recent years. In engineering and natural sciences, the nature of a dynamic system is determined by the accuracy of the knowledge we have about the parameters that describe it. A deterministic dynamical system occurs when knowledge about a dynamic system is precise. To put it another way, evaluating the parameters of a dynamic system is not without risk. When our knowledge of the parameters of a dynamic system is statistical in nature, or when the information is probabilistic, differential equations or stochastic differential equations are commonly used in mathematical modeling of such systems.

Our prior work investigates the existence of mild solution for controllability of nonlocal impulsive differential equations via measure of noncompactness, see [37]. The measure of noncompactness property and Darbo-Sadovskii's fixed point theorem are used to determine the controllability of nonlocal impulsive differential equations under Lipschitz 
conditions. Next, we investigated the existence and uniqueness of the controllability of neutral impulsive differential equations with nonlocal conditions in prior work. The controllability of a nonlocal neutral impulsive differential equation under compactness conditions, Lipschitz conditions, and mixed-type conditions is obtained using the property of measure of noncompactness and Darbo-Sadovskii's fixed point theorem, read the cited papers [38,39]. We now extend our research to investigate the controllability and wellposedness of an impulsive functional abstract second order differential equation with state dependent delay. The study of controllability and wellposedness of impulsive functional abstract second-order differential equations with state dependent delay is a nearly unexplored area in the literature, which is the motivating factor behind this paper.

By using the ideas of cited papers [25,40], we investigate that problem (1)-(4) is controllable and also show the wellposedness of the problem (1)-(4). We use this fact in Theorem 1 that shows the controllability condition in $C_{L i p}^{1}([-\gamma, b] ; X)$ exists. The wellposedness of the problem (1)-(4) is proved in Proposition 1. Consider the condition $\sigma_{j}(0, \varphi)=0, j=1,2$ from the above results. The term $\mathscr{F}\left(t, y_{\sigma_{1}\left(t, y_{t}\right)}, y_{\sigma_{2}\left(t, y_{t}\right)}^{\prime}\right)$ act as class of equations and it is represented as $\mathscr{G}\left(t, y\left(\rho_{1}\left(t, y_{t}\right)\right), y^{\prime}\left(\rho_{2}\left(t, y_{t}\right)\right)\right)$. The instance $\rho(0, \varphi)<0$ is studied for the above cases. Finally, we illustrate few applications on impulsive differential equations of second order with delay condition.

\section{Basic Preliminaries}

The cosine family $(C(t))_{t \in \mathbb{R}}$ is defined in $X$ and the norm $\|y\|_{\mathscr{A}}=\|x\|+\|\mathscr{A} x\|$ is described from the domain $\mathfrak{D}$ of $\mathscr{A}$. The sine function $(S(t))_{t \in \mathbb{R}}$ is associated to $(C(t))_{t \in \mathbb{R}}$ defined by $S(t) x:=\int_{0}^{t} C(s) x d s$, for $x \in X$ and $t \in \mathbb{R}$. Furthermore, $C_{0}$ is a non-negative constant and it's norm described as $\|C(t)\| \leq C_{0}$, for every $t \in[0, b]$.

Here, we describe the phase space $\mathscr{B}$ axiomatically, using notions and annotations prepared in [41] and suitably modified to the impulsive differential equations. More particularly, $\mathscr{B}:(-\infty, 0] \rightarrow X$ denotes the vector space of functions provided with a norm denoted as $\|\cdot\|_{\mathscr{B}}$ and thus, the following axioms hold:

(I) If $y:(-\infty, \mu+a] \rightarrow X, a>0$, is a function such that $y_{\mu} \in \mathscr{B}$ and $\left.x\right|_{[\mu, \mu+a]} \in$ $C([\mu, \mu+a], X)$, then, for every $t \in[\mu, \mu+a)$, the following properties hold:

(i) $y_{t}$ is in $\mathscr{B}$,

(ii) $\|y(t)\| \leq H\left\|y_{t}\right\|_{\mathscr{B}}$,

(iii) $\left\|y_{t}\right\|_{\mathscr{B}} \leq K_{1}(t-\mu) \sup \|y(s)\|_{\mathbb{X}}: \mu \leq s \leq t+K_{2}(t-\mu)\left\|y_{\mu}\right\|$, where $H>0$ is a constant; $K_{1}, K_{2}:[0, \infty) \rightarrow[1, \infty), K_{1}$ is continuous, $K_{2}$ is locally bounded and $H, K_{1}, K_{2}$ are independent of $y(\cdot)$.

(II) The space $\mathscr{B}$ is complete.

The space $\mathcal{E}=\{x \in X: C(\cdot) x$ is continuosly differentiable $\}$ awarded with the norm $\|x\|_{\mathcal{E}}=\|x\|+\sup _{0 \leq t \leq b}\|\mathscr{A} S(t) x\|$. From the literature of Kisiński [42], since $\mathcal{E}$ is a Banach space, $\mathscr{A} S(t) \in \mathscr{L}(\mathcal{E}, X)$, for every $t$ in the real line $\mathbb{R}$ and if $s$ converges to 0 then $\mathscr{A} S(s) x$ converges to 0 , for every $x \in \mathcal{E}$.

Now, We define the abstract differential equation of second order

$$
\begin{gathered}
u^{\prime \prime}(t)=\mathscr{A} u(t)+\eta(t), \quad t \in[0, b], \\
u(0)=x_{1}, \quad u^{\prime}(0)=x_{2} .
\end{gathered}
$$

Here, $\eta$ is an integrable function defined from $[0, b]$ to $X$ and $x_{1}, x_{2} \in X$. The mild solution of (5) and (6) is described as

$$
u(t)=C(t) x_{1}+S(t) x_{2}+\int_{0}^{t} S(t-s) \eta(s) d s, \quad t \in[0, b],
$$


and for $x_{1} \in \mathcal{E}$, the $C^{1}$ function $u(\cdot)$ defined on $[0, b]$,

$$
u^{\prime}(t)=\mathscr{A} S(t) x_{1}+C(t) x_{2}+\int_{0}^{t} C(t-s) \eta(s) d s, t \in[0, b] .
$$

We study the further feature on abstract Cauchy problem of second order and Cosine functions from the reference of the papers $[43,44]$. Let $C([d, e] ; X)$ and $C_{L i p}([d, e] ; X)$ be normed spaces and it's norms are denoted as $\|\cdot\|_{C([d, e] ; X)}$ and $\|\cdot\|_{C_{L i p}([d, e] ; X)}$, respectively. We termed that

$$
\|\cdot\|_{C_{L i p}([d, e] ; X)}=\|\cdot\|_{C([d, e] ; X)}+[\cdot]_{C_{L i p}([d, e] ; X)},
$$

where, $[\zeta]_{C_{L i p}([d, e] ; X)}=\sup _{t, s \in[d, e], t \neq s} \frac{\|\zeta(s)-\zeta(t)\|_{X}}{|t-s|}$. The space $C_{L i p}^{1}([d, e] ; X)$ is constructed for all $\epsilon \in C_{L i p}([d, e] ; X)$ such that $\epsilon^{\prime} \in C_{L i p}([d, e] ; X)$, associated with the norm $\|\epsilon\|_{C_{L i p}^{1}([d, e] ; X)}$ $=\|\epsilon\|_{C_{L i p}([d, e] ; X)}+\left\|\epsilon^{\prime}\right\|_{C_{L i p}([d, e] ; X)}$.

We use the state-dependent delay from [25]. The function $y_{(\cdot)}:[0, a] \rightarrow \mathscr{B}$ noted as $y_{(\cdot)}$ is defined by $y_{(\cdot)}(s)=y_{s}$, for $y \in C([-\gamma, a] ; X)$ and $0<a \leq b$. This consequence and the Lemma 1 are used for our main results of the article.

Lemma 1 ([25] Lemma 1). Suppose $y, z \in C([-\gamma, a] ; X), 0<a \leq b$, the function $\sigma(\cdot)$ belongs to $C_{\text {Lip }}\left([0, b] \times \mathscr{B} ; \mathbb{R}^{+}\right), u_{\mid[0, a]}, v_{\mid[0, a]} \in C_{L i p}([0, a] ; X), u_{0}=\omega, v_{0}=\phi$, for $\omega, \phi \in$ $C_{L i p}([-\gamma, 0] ; X)$, and $\sigma\left(t, h_{t}\right) \leq a$, for $\eta=y, z$ and every $t \in[0, a]$. Then $y_{(\cdot)}, y_{\sigma(\cdot, u(\cdot)} \in$ $C_{\text {Lip }}([0, a] ; \mathscr{B}),\left[y_{(\cdot)}\right]_{C_{\text {Lip }}([0, a] ; \mathscr{B})} \leq \max \left\{[y]_{C_{\text {Lip }}([0, a] ; X)},[\omega]_{C_{\text {Lip }}([-\gamma, 0] ; X)}\right\}$ and

$$
\begin{aligned}
{\left[y_{\sigma(\cdot, y(\cdot)}\right]_{C_{L i p}([0, a] ; \mathscr{B})} } & \leq\left[y_{(\cdot)}\right]_{C_{L i p}([0, a] ; \mathscr{B})}[\sigma]_{C_{L i p}\left([0, a] \times \mathscr{B} ; \mathbb{R}^{+}\right)}\left(1+\left[y_{(\cdot)}\right]_{C_{L i p}([0, a] ; \mathscr{B})}\right) \\
\| y_{\sigma\left(\cdot, y_{(\cdot)}\right)}-z_{\sigma(\cdot, z(\cdot))} & \|_{C([0, a] ; \mathscr{B})} \\
& \leq\left(1+\left[z_{(\cdot)}\right]_{C_{L i p}([0, a] ; \mathscr{B})}[\sigma]_{C_{L i p}\left([0, a] \times \mathscr{B} ; \mathbb{R}^{+}\right)}\right)\|y-z\|_{C([-\gamma, a] ; X)} .
\end{aligned}
$$

Moreover, $y, z \in C_{L i p}^{1}([-\gamma, a] ; X)$, then $y_{\sigma(\cdot, y(\cdot)}^{\prime} \in C_{L i p}([0, a] ; \mathscr{B})$ and

$$
\begin{aligned}
{\left[y_{\sigma(\cdot, y(\cdot)}^{\prime}\right]_{C_{L i p}([0, a] ; \mathscr{B})} } & \leq\left[y_{(\cdot)}^{\prime}\right]_{C_{L i p}([0, a] ; \mathscr{B})}[\sigma]_{C_{L i p}\left([0, a] \times \mathscr{B} ; \mathbb{R}^{+}\right)}\left(1+\left[y_{(\cdot)}\right]_{C_{L i p}([0, a] ; \mathscr{B})}\right) \\
\| y_{\sigma(\cdot, y(\cdot)}^{\prime}-z_{\sigma\left(\cdot, z_{(\cdot)}\right)}^{\prime} & \|_{C([0, a] ; \mathscr{B})} \\
& \leq\left(1+\left[y_{(\cdot)}^{\prime}\right]_{C_{L i p}([0, a] ; \mathscr{B})}[\sigma]_{C_{L i p}\left([0, a] \times \mathscr{B} ; \mathbb{R}^{+}\right)}\right)\|y-z\|_{C^{1}([-\gamma, a] ; X)} .
\end{aligned}
$$

Lemma 2 ([4], Sadovskii's Fixed Point Theorem). Let E be a condensing operator on a Banach space $X$. If $E(S) \subset S$ for a convex, closed and bounded set $S$ of $X$, then $E$ has a fixed point in $S$.

\section{Main Results}

First, the existence of controllability of the problem (1)-(4) is studied. We define the mild solution and controllable of the problem (1)-(4). Then we prove the wellposedness of problem (1)-(4). In view of simplification, suppose that $\sigma_{1}(\cdot)=\sigma_{2}(\cdot)$ and we termed that $\|\cdot\|_{\mathscr{B}}$ for $\|\cdot\|_{C([-\gamma, 0] ; X)}$. In our argument, we take $\sigma_{1} \neq \sigma_{2}$ as in the remaining of this work. We present following definitions and assumptions for further study.

Definition 1. The function $y \in C^{1}([0, a] ; X)$ is mild solution of the problem (1)-(4) on $[-\gamma, a]$ defined by

$y(t)=C(t) \varphi(0)+S(t) x+\int_{0}^{t} S(t-s)\left[B u(s)+\mathscr{F}\left(s, y_{\sigma\left(s, y_{s}\right)}, y_{\sigma\left(s, y_{s}\right)}^{\prime}\right)\right] d s+\sum_{0<t_{k}<t} C\left(t-t_{k}\right) I_{k}\left(y_{t_{k}}\right)+\sum_{0<t_{k}<t} S\left(t-t_{k}\right) J_{k}\left(y_{t_{k}}\right)$,

for all $t \in[0, a], 0<a \leq b$ and if $y_{0}=\varphi$.

Following conditions are useful to prove the main results. 
$\left(H_{\sigma, \varphi}\right) \quad \sigma \in C_{L i p}\left([0, b] \times \mathscr{B} ; \mathbb{R}^{+}\right), \sigma(0, \varphi)=0$ and a non-negative number $r^{*}$ exists and $0<$ $a^{*} \leq b$ provides $0 \leq \sigma(t, \omega) \leq t$, for every $t \in\left[0, a^{*}\right]$ and $\omega \in B_{r^{*}}(\varphi, \mathscr{B})$.

$\left(H_{I, J}\right) \quad$ There exists a positive constants $L_{I_{k}}, L_{J_{k}}$ such that

$$
\begin{aligned}
& \left\|I_{k}\left(\omega_{1}\right)-I_{k}\left(\omega_{2}\right)\right\| \leq L_{I_{k}}\left\|\omega_{1}-\omega_{2}\right\|_{\mathscr{B}}, \omega_{j} \in \mathscr{B}, j=1,2, \quad k=1,2, \ldots, n, \\
& \left\|J_{k}\left(\omega_{1}\right)-J_{k}\left(\omega_{2}\right)\right\| \leq L_{J_{k}}\left\|\omega_{1}-\omega_{2}\right\|_{\mathscr{B}}, \omega_{j} \in \mathscr{B}, j=1,2, \quad k=1,2, \ldots, n .
\end{aligned}
$$

$\left(H_{W}\right) \quad$ If $B: U \rightarrow X$ is a continuous operator and the operator $W: \mathscr{L}^{2}([0, b], U) \rightarrow X$ is linear, described by

$$
W u=\int_{0}^{b} S(b-s) B u(s) d s,
$$

has a bounded invertible operator $W^{-1}$ provided $\|B\| \leq \mathfrak{M}_{1}$ and $\left\|W^{-1}\right\| \leq \mathfrak{M}_{2}$, for some positive constant $\mathfrak{M}_{1}, \mathfrak{M}_{2}$.

$\left(H_{\Phi, \Psi}\right) \quad$ Functions $I_{k}, J_{k}: \mathscr{B} \rightarrow X, k=1,2, \cdots, n$ are continuous completely and there exist functions $\Phi_{k}, \Psi_{k}:[0, \infty) \rightarrow(0, \infty), k=1,2, \cdots, n$ which are continuous and non decreasing such that

$$
\begin{aligned}
\left\|I_{k}(\omega)\right\| \leq \Phi_{k}\left(\|\omega\|_{\mathscr{B}}\right), & & \liminf _{\zeta \rightarrow+\infty} \frac{\Phi_{k}(\varsigma)}{\zeta}=\varsigma_{k}<\infty . \\
\left\|J_{k}(\omega)\right\| \leq \Psi_{k}\left(\|\omega\|_{\mathscr{B}}\right), & & \liminf _{\zeta \rightarrow+\infty} \frac{\Psi_{k}(\varsigma)}{\varsigma}=\eta_{k}<\infty .
\end{aligned}
$$

Definition 2. The system (1)-(4) is said to be controllable on the interval $[0, b]$, if for every $\phi \in \mathcal{D}(\mathscr{A})$ and $y_{1} \in X$, there exists a control $u \in \mathscr{L}^{2}([0, b], U)$ such that the mild solution function $y(t)$ of (1)-(4) satisfies $y(b)=y_{1}$.

Remark 1. Also, we define the following constants for our convenience

$$
K_{b}=\|y(b)\|+C_{0}\|\varphi(0)\|+C_{0} a\left\|\varphi^{\prime}(0)\right\|+C_{0} a b\left(2 L_{F}+\sup _{\tau \in[0, b]}\left\|\mathscr{F}\left(\tau, \varphi, \varphi^{\prime}\right)\right\|\right)+C_{0} \sum_{k=1}^{n}\left(\Phi_{k}\left(r^{*}\right)+a \Psi_{k}(r *)\right) .
$$

Now, we can obtain the first main result.

Theorem 1. Suppose $\left(H_{\sigma, \varphi}\right),\left(H_{I, J}\right),\left(H_{W}\right)$ and $\left(H_{\Phi, \Psi}\right)$ holds. Also, $\varphi \in C_{L i p}^{1}([-\gamma, 0] ; X)$, $C(\cdot) \varphi(0), \mathscr{A} S(\cdot) \varphi(0), C(\cdot) \varphi^{\prime}(0) \in C_{\text {Lip }}([0, a] ; X)$ and $\mathscr{F} \in C_{\text {Lip }}([0, b] \times \mathscr{B} \times \mathscr{B} ; X)$ holds. Then the system (1)-(4) is controllable on $[-\gamma, a]$, for any $0<a \leq b$.

Proof. Using the hypothesis $\left(H_{W}\right)$, we define the control function in terms of $y(\cdot)$,

$$
u(t)=W^{-1}\left\{y(b)-C(b) \varphi(0)-S(b) \varphi^{\prime}(0)-\int_{0}^{b} S(b-s) \mathscr{F}\left(s, y_{\sigma\left(s, y_{s}\right)}, y_{\sigma\left(s, y_{s}\right)}^{\prime}\right) d s-\sum_{k=1}^{n} C\left(b-t_{k}\right) I_{k}\left(y_{t_{k}}\right)-\sum_{k=1}^{n} S\left(b-t_{k}\right) J_{k}\left(y_{t_{k}}\right)\right\}(t) .
$$

Let $d^{*}$ and $r^{*}$ be defined in condition $\left(H_{\sigma, \varphi}\right)$ and the Equation (13) holds. Let $\mathcal{R}>0$ be sufficiently large number satisfying

$$
\begin{aligned}
& \mathcal{R}>[\varphi]_{C_{L i p}^{1}([-\gamma, 0] ; X)}+[C(\cdot) \varphi(0)]_{C_{L i p}([0, b] ; X)}+[\mathscr{A} S(\cdot) \varphi(0)]_{C_{L i p}([0, b] ; X)}+\left[C(\cdot) \varphi^{\prime}(0)\right]_{C_{L i p}([0, b] ; X)}+C_{0}\left\|\varphi^{\prime}(0)\right\| \\
& +4 C_{0}\left(2 L_{F}+\sup _{\tau \in[0, b]}\left\|\mathscr{F}\left(\tau, \varphi, \varphi^{\prime}\right)\right\|\right)+3 C_{0} a \mathfrak{M}_{1} \mathfrak{M}_{2} K_{b}+\sum_{k=1}^{n}\left([\mathscr{A} S(\cdot)]_{C_{L i p}([0, b] ; X)}+C_{0}\right) \Psi_{k}(r *), k=1,2, \cdots, n .
\end{aligned}
$$


Let us assume the condition $\mathcal{R} a \leq \min \left\{b, d^{*}, r^{*}, 1\right\}$ when $0<a<\min \left\{b, d^{*}, 1\right\}$ and for $k=1,2, \cdots, n$

$$
\begin{aligned}
& \Lambda=C_{0}\left[2 a(1+a)\left(1+C_{0} a b \mathfrak{M}_{1} \mathfrak{M}_{2}\right) L_{F}\left(1+\mathcal{R}[\sigma]_{C_{L i p}\left([0, b] \times \mathscr{B} ; \mathbb{R}^{+}\right)}\right)+a(1+a) \mathfrak{M}_{1} \mathfrak{M}_{2}\right. \\
&\left.+\left(1+2 C_{0} a \mathfrak{M}_{1} \mathfrak{M}_{2}\right) \sum_{k=1}^{n}\left(L_{I_{k}}+a L_{J_{k}}\right)+\sum_{k=1}^{n}\left(\mathscr{A} a L_{I_{k}}+L_{J_{k}}\right)\right]<1 \\
& {[C(\cdot) \varphi(0)]_{C_{L i p}([0, b] ; X)}+[\mathscr{A} S(\cdot) \varphi(0)]_{C_{L i p}([0, b] ; X)}+\left[C(\cdot) \varphi^{\prime}(0)\right]_{C_{L i p}([0, b] ; X)}+C_{0}\left\|\varphi^{\prime}(0)\right\| } \\
&+4 C_{0}\left(2 L_{F}+\sup _{\tau \in[0, b]}\left\|\mathscr{F}\left(\tau, \varphi, \varphi^{\prime}\right)\right\|\right)+a C_{0} L_{F}\left(1+2 \mathcal{R}[\sigma]_{C_{L i p}\left([0, b] \times \mathscr{B} ; \mathbb{R}^{+}\right)}(1+\mathcal{R})\right) \\
&+3 C_{0} a \mathfrak{M}_{1} \mathfrak{M}_{2} K_{b}+\sum_{j=1}^{n}\left([\mathscr{A} S(\cdot)]_{C_{L i p}([0, b] ; X)}+C_{0}\right) \Psi_{k}(r *)+\epsilon \leq \mathcal{R} .
\end{aligned}
$$

Let $\mathcal{Y}(a, \mathcal{R})=\left\{y \in C([-\gamma, a] ; X): y_{0}=\varphi, \max \left\{[y]_{C_{L i p}([-\gamma, a] ; X)},\left[y^{\prime}\right]_{C_{L i p}([-\gamma, a] ; X)} \leq\right.\right.$ $\mathcal{R}\}$ provided that $d(y, z)=\|y-z\|_{C^{1}([0, a] ; X)}$ and the function $\mathcal{G}: \mathcal{Y}(a, \mathcal{R}) \rightarrow C([-\gamma, a] ; X)$ given by $\mathcal{G} y(t)=\varphi(t)$ for $t \in[-\gamma, 0]$, we have

$$
\begin{aligned}
\mathcal{G} y(t)= & C(t) \varphi(0)+S(t) \varphi^{\prime}(0)+\int_{0}^{t} S(t-s) \mathscr{F}\left(s, y_{\sigma\left(s, y_{s}\right)}, y_{\sigma\left(s, y_{s}\right)}^{\prime}\right) d s+\int_{0}^{t} S(t-\xi) B W^{-1}\left\{y(b)-C(b) \varphi(0)-S(b) \varphi^{\prime}(0)\right. \\
& \left.-\int_{0}^{b} S(b-s) \mathscr{F}\left(s, y_{\sigma\left(s, y_{s}\right)}, y_{\sigma\left(s, y_{s}\right)}^{\prime}\right) d s-\sum_{k=1}^{n} C\left(b-t_{k}\right) I_{k}\left(y_{t_{k}}\right)-\sum_{k=1}^{n} S\left(b-t_{k}\right) J_{k}\left(y_{t_{k}}\right)\right\}(\xi) d \xi \\
& +\sum_{0<t_{k}<t} C\left(t-t_{k}\right) I_{k}\left(y_{t_{k}}\right)+\sum_{0<t_{k}<t} S\left(t-t_{k}\right) J_{k}\left(y_{t_{k}}\right),
\end{aligned}
$$

for $t \in[0, a]$. Let $y \in \mathcal{Y}(a, \mathcal{R})$. By Lemma 1 and the selection of $\mathcal{R}$, we have

$$
\left\|y_{t}-\varphi\right\|_{\mathscr{B}} \leq\left[y_{(\cdot)}\right]_{C_{L i p}([0, a] ; \mathscr{B})} a \leq \max \left\{[y]_{C_{L i p}([0, a] ; X)},[\varphi]_{C_{L i p}([-\gamma, 0] ; X)}\right\} a \leq \mathcal{R} a \leq r^{*},
$$

which gives $0 \leq \sigma\left(t, y_{t}\right) \leq t \leq a$, for every $t \in[0, a]$. Which shows that $y_{\sigma\left(\cdot, y_{(\cdot)}\right)}, y_{\sigma(\cdot, y(\cdot))^{\prime}}^{\prime}$ $\mathscr{F}\left(\cdot, y_{\sigma\left(\cdot, y_{(\cdot)}\right)}, y_{\sigma\left(\cdot, y_{(\cdot)}\right)}^{\prime}\right)$ and $\mathcal{G} y(\cdot)$ are well defined. Moreover, since $\sigma\left(t, y_{t}\right) \leq t \leq a$ and $\max \left\{\left\|y_{\sigma\left(t, y_{t}\right)}-\varphi\right\|_{\mathscr{B}},\left\|y_{\sigma\left(t, y_{t}\right)}^{\prime}-\varphi^{\prime}\right\|_{\mathscr{B}} \|\right\} \leq \mathcal{R} a \leq 1$, for every $t \in[0, a]$. Using this, we obtain that

$$
\begin{aligned}
\left\|\mathscr{F}\left(t, y_{\sigma\left(t, y_{t}\right)}, y_{\sigma\left(t, y_{t}\right)}^{\prime}\right)\right\| & \leq\left\|\mathscr{F}\left(t, y_{\sigma\left(t, y_{t}\right)}, y_{\sigma\left(t, y_{t}\right)}^{\prime}\right)-\mathscr{F}\left(t, \varphi, \varphi^{\prime}\right)\right\|+\left\|\mathscr{F}\left(t, \varphi, \varphi^{\prime}\right)\right\| \\
& \leq L_{F}\left(\left\|y_{\sigma\left(t, y_{t}\right)}-\varphi\right\|+\left\|y_{\sigma\left(t, y_{t}\right)}^{\prime}-\varphi^{\prime}\right\|\right)+\sup _{\tau \in[0, a]}\left\|\mathscr{F}\left(\tau, \varphi, \varphi^{\prime}\right)\right\| \\
& \leq 2 L_{F}+\sup _{\tau \in[0, a]}\left\|\mathscr{F}\left(\tau, \varphi, \varphi^{\prime}\right)\right\| .
\end{aligned}
$$

Since the map $I_{k}$ is continuous completely and $(C(t))_{t \in \mathbb{R}}$ is continuous strongly; for given $\epsilon>0$, there is a number $\delta>0$ implies

$$
\left\|[C(t+\eta)-C(t)] I_{k}(\Phi)\right\| \leq \frac{\epsilon}{n}, k=1, \cdots, n .
$$

Using (17) and (18), for all $t \in[0, a)$ and $t+\eta \in[0, a]$, for $\eta>0$, we have 
$\|\mathcal{G} y(t+\eta)-\mathcal{G} y(t)\|$

$\leq[C(\cdot) \varphi(0)]_{C_{L i p}([0, a] ; X)} \eta+C_{0}\left\|\varphi^{\prime}(0)\right\| \eta+\int_{0}^{t}\|S(s+\eta)-S(s)\|\left\|\mathscr{F}\left(s, y_{\sigma\left(s, y_{s}\right)}, y_{\sigma\left(t, y_{t}\right)}^{\prime}\right)\right\| d s$

$+\int_{t}^{t+\eta}\left\|S(t+\eta-s) \mathscr{F}\left(s, y_{\sigma\left(s, y_{s}\right)}, y_{\sigma\left(t, y_{t}\right)}^{\prime}\right)\right\| d s$

$+\int_{0}^{t} \|[S(\xi+\eta)-S(\xi)] B W^{-1}\left\{y(b)-C(b) \varphi(0)-S(b) \varphi^{\prime}(0)\right.$

$\left.-\int_{0}^{b} S(b-s) \mathscr{F}\left(s, y_{\sigma\left(s, y_{s}\right)}, y_{\sigma\left(s, y_{s}\right)}^{\prime}\right) d s-\sum_{k=1}^{n} C\left(b-t_{k}\right) I_{k}\left(y_{t_{k}}\right)-\sum_{k=1}^{n} S\left(b-t_{k}\right) J_{k}\left(y_{t_{k}}\right)\right\}(\xi) d \xi \|$

$+\int_{t}^{t+\eta} \| S(t+\eta-\xi) B W^{-1}\left\{y(b)-C(b) \varphi(0)-S(b) \varphi^{\prime}(0)\right.$

$\left.-\int_{0}^{b} S(b-s) \mathscr{F}\left(s, y_{\sigma\left(s, y_{s}\right)}, y_{\sigma\left(s, y_{s}\right)}^{\prime}\right) d s-\sum_{k=1}^{n} C\left(b-t_{k}\right) I_{k}\left(y_{t_{k}}\right)-\sum_{k=1}^{n} S\left(b-t_{k}\right) J_{k}\left(y_{t_{k}}\right)\right\}(\xi) d \xi \|$

$+\sum_{k=1}^{n}\left\|\left[C\left(t+\eta-t_{k}\right)-C\left(t-t_{k}\right)\right] I_{k}\left(y_{t_{k}}\right)\right\|+\sum_{k=1}^{n}\left\|\left[S\left(t+\eta-t_{k}\right)-S\left(t-t_{k}\right)\right] J_{k}\left(y_{t_{k}}\right)\right\|$

$\leq[C(\cdot) \varphi(0)]_{C_{L i p}([0, a] ; X)} \eta+C_{0}\left\|\varphi^{\prime}(0)\right\| \eta+2 C_{0} \eta \sup _{s \in[0, a]}\left\|\mathscr{F}\left(s, y_{\sigma\left(s, y_{s}\right)}, y_{\sigma\left(t, y_{t}\right)}^{\prime}\right)\right\| a$

$+C_{0} \eta \mathfrak{M}_{1} \mathfrak{M}_{2}\left[\|y(b)\|+C_{0}\|\varphi(0)\|+C_{0} a\left\|\varphi^{\prime}(0)\right\|+C_{0} a b \sup _{s \in[0, b]}\left\|\mathscr{F}\left(s, y_{\sigma\left(s, y_{s}\right)}, y_{\sigma\left(s, y_{s}\right)}^{\prime}\right)\right\|\right.$

$\left.+C_{0} \sum_{k=1}^{n}\left\|I_{k}\left(y_{t_{k}}\right)\right\|+C_{0} a \sum_{k=1}^{n}\left\|J_{k}\left(y_{t_{k}}\right)\right\|\right] a+C_{0} a \mathfrak{M}_{1} \mathfrak{M}_{2}\left[\|y(b)\|+C_{0}\|\varphi(0)\|+C_{0} a\left\|\varphi^{\prime}(0)\right\|\right.$

$\left.+C_{0} a b \sup _{s \in[0, b]}\left\|\mathscr{F}\left(s, y_{\sigma\left(s, y_{s}\right)}, y_{\sigma\left(t, y_{t}\right)}^{\prime}\right)\right\|+C_{0} \sum_{k=1}^{n}\left\|I_{k}\left(y_{t_{k}}\right)\right\|+C_{0} a \sum_{k=1}^{n}\left\|J_{k}\left(y_{t_{k}}\right)\right\|\right] \eta+\epsilon+C_{0} \eta \sum_{k=1}^{n}\left(\Psi_{k}(r *)\right)$

$\leq[C(\cdot) \varphi(0)]_{C_{L i p}([0, a] ; X)} \eta+C_{0}\left\|\varphi^{\prime}(0)\right\| \eta+2 a C_{0}\left(2 L_{F}+\sup _{\tau \in[0, a]}\left\|\mathscr{F}\left(\tau, \varphi, \varphi^{\prime}\right)\right\|\right) \eta$

$+2 a C_{0} \eta \mathfrak{M}_{1} \mathfrak{M}_{2} K_{b}+C_{0} \eta \sum_{k=1}^{n}\left(\Psi_{k}(r *)\right)+\epsilon$,

since the Equation (15), $[\mathcal{G} y]_{C_{L i p}([0, a] ; X)} \leq \mathcal{R}$. We know that $(\mathcal{G} y)_{0}=\varphi, \varphi \in C_{L i p}([-\gamma, 0] ; X)$ and $\mathcal{R}>[\varphi]_{\mathcal{C}_{L i p}([-\gamma, 0] ; X)}$, from Lemma 1 , we obtain that $\mathcal{G} y \in C_{L i p}([-\gamma, a] ; X)$ and $[\mathcal{G} y]_{C_{L i p}([-\gamma, a] ; X)} \leq \mathcal{R}$.

To evaluate $\left[(\mathcal{G} y)^{\prime}\right]_{C_{L i p}([-\gamma, a] ; X)}$, the $C^{1}$ function $\mathcal{G} y(\cdot)$ is observed and

$$
\begin{aligned}
(\mathcal{G} y)^{\prime}(t)= & \mathscr{A} S(t) \varphi(0)+C(t) \varphi^{\prime}(0)+\int_{0}^{t} C(t-s) \mathscr{F}\left(s, y_{\sigma\left(s, y_{s}\right)}, y_{\sigma\left(s, y_{s}\right)}^{\prime}\right) d s+\int_{0}^{t} C(t-\xi) B W^{-1}(y(b)-C(b) \varphi(0) \\
& \left.-S(b) \varphi^{\prime}(0)-\int_{0}^{b} S(b-s) \mathscr{F}\left(s, y_{\sigma\left(s, y_{s}\right)}, y_{\sigma\left(s, y_{s}\right)}^{\prime}\right) d s-\sum_{0<t_{k}<t} C\left(b-t_{k}\right) I_{k}\left(y_{t_{k}}\right)-\sum_{0<t_{k}<t} S\left(b-t_{k}\right) J_{k}\left(y_{t_{k}}\right)\right) d s \\
& +\sum_{0<t_{k}<t} \mathscr{A} S\left(t-t_{k}\right) I_{k}\left(y_{t_{k}}\right)+\sum_{0<t_{k}<t} C\left(t-t_{k}\right) J_{k}\left(y_{t_{k}}\right), \text { for } t \in[0, a] .
\end{aligned}
$$

Simply, we have $\mathscr{F}\left(\cdot, y_{\sigma\left(\cdot, y_{(\cdot)}\right)}, y_{\sigma(\cdot, y(\cdot))}^{\prime}\right) \in C_{L i p}([0, a] ; X)$ by Lemma 1 , and

$$
\begin{aligned}
& \left.\left[\mathscr{F}\left(\cdot, y_{\sigma(\cdot, y(\cdot)}\right), y_{\sigma\left(\cdot, y_{(\cdot)}^{\prime}\right)}^{\prime}\right)\right]_{C_{L i p}([0, a] ; X)} \\
& \quad \leq \quad L_{F}\left(1+\left(\left[y_{(\cdot)}\right]_{C_{L i p}([0, a] ; \mathscr{B})}+\left[y_{(\cdot)}^{\prime}\right]_{C_{L i p}([0, a] ; \mathscr{B})}\right)\right)[\sigma]_{C_{L i p}\left([0, b] \times \mathscr{B} ; \mathbb{R}^{+}\right)}\left(1+\left[y_{(\cdot)}\right]_{C_{L i p}([0, a] ; \mathscr{B})}\right) \\
& \quad \leq \quad L_{F}\left(1+2 \mathcal{R}[\sigma]_{C_{L i p}\left([0, b] \times \mathscr{B} ; \mathbb{R}^{+}\right)}(1+\mathcal{R})\right) .
\end{aligned}
$$


Using (18) and (19), as $t \in[0, a)$ and $\eta>0$ over $t+\eta \in[0, a]$, it is noted that

$$
\begin{aligned}
& \left\|(\mathcal{G} y)^{\prime}(t+\eta)-(\mathcal{G} y)^{\prime}(t)\right\| \\
& \leq \quad[\mathscr{A} S(\cdot) \varphi(0)]_{C_{L i p}([0, a] ; X)} \eta+\left[C(\cdot) \varphi^{\prime}(0)\right]_{C_{L i p}([0, a] ; X)} \eta+C_{0}\left(2 L_{F}+\sup _{\tau \in[0, b]}\left\|\mathscr{F}\left(\tau, \varphi, \varphi^{\prime}\right)\right\|\right) \eta \\
& \quad+C_{0} \eta \mathfrak{M}_{1} \mathfrak{M}_{2} K_{b}+a C_{0} L_{F}\left(1+2 \mathcal{R}[\sigma]_{C_{L i p}\left([0, b] \times \mathscr{B} ; \mathbb{R}^{+}\right)}(1+\mathcal{R})\right) \eta+\sum_{k=1}^{n}\left[\mathscr{A} S(\cdot)\left(\Psi_{k}(r *)\right)\right]_{C_{L i p}([0, a] ; X)} \eta+\epsilon .
\end{aligned}
$$

Hence, $\left[(\mathcal{G} y)^{\prime}\right]_{C_{L i p}([0, a] ; X)} \leq \mathcal{R}$. In addition, since $\frac{d^{+} y}{d t}(0)=\varphi^{\prime}(0)=\frac{d^{-} y}{d t}(0)=\frac{d^{-} \varphi}{d t}(0)$ and $\mathcal{R}>\left[\varphi^{\prime}\right]_{C_{L i p}([-\gamma, 0] ; X)}$, we conclude that $\left[(\mathcal{G} y)^{\prime}\right] \in C([-\gamma, a] ; X)$ and $\left[(\mathcal{G} y)^{\prime}\right]_{C_{L i p}([-\gamma, a] ; X)}$ $\leq \mathcal{R}$. Which shows that $\mathcal{G}$ is a $\mathcal{Y}(a, \mathcal{R})$-valued function.

A contrary result obtained from Lemma 1 and for every $y, z \in \mathscr{Y}(a, \mathcal{R}), t \in[0, a]$ gives

$$
\begin{aligned}
\|\mathcal{G} y(t)-\mathcal{G} z(t)\| \leq & \int_{0}^{t} C_{0} a L_{F}\left(\left\|y_{\sigma\left(s, y_{s}\right)}-z_{\sigma\left(s, z_{s}\right)}\right\| \mathscr{B}+\left\|y_{\sigma\left(s, y_{s}\right)}^{\prime}-z_{\sigma\left(s, z_{s}\right)}^{\prime}\right\| \mathscr{B}\right) d s \\
& +\int_{0}^{t} C_{0} a \mathfrak{M}_{1} \mathfrak{M}_{2}\left[\|y(b)-z(b)\|+\int_{0}^{b} C_{0} a L_{F}\left(\left\|y_{\sigma\left(s, y_{s}\right)}-z_{\sigma\left(s, z_{s}\right)}\right\|_{\mathscr{B}}+\left\|y_{\sigma\left(s, y_{s}\right)}^{\prime}-z_{\sigma\left(s, z_{s}\right)}^{\prime}\right\|_{\mathscr{B}}\right) d s\right. \\
& \left.+C_{0} \sum_{k=1}^{n}\left\|I_{k}\left(y\left(t_{k}\right)\right)-I_{k}\left(z\left(t_{k}\right)\right)\right\|+C_{0} a \sum_{k=1}^{n}\left\|J_{k}\left(y\left(t_{k}\right)\right)-J_{k}\left(z\left(t_{k}\right)\right)\right\|\right](\xi) d \xi \\
& +C_{0} \sum_{k=1}^{n}\left\|I_{k}\left(y\left(t_{k}\right)\right)-I_{k}\left(z\left(t_{k}\right)\right)\right\|+C_{0} a \sum_{k=1}^{n}\left\|J_{k}\left(y\left(t_{k}\right)\right)-J_{k}\left(z\left(t_{k}\right)\right)\right\| \\
\leq & 2 C_{0} a^{2} L_{F}\left(1+C_{0} a b \mathfrak{M}_{1} \mathfrak{M}_{2}\right)\left(1+\left[z_{(\cdot)}\right]_{C_{L i p}([0, a] ; \mathscr{B})}[\sigma]_{C_{L i p}\left([0, b] \times \mathscr{B} ; \mathbb{R}^{+}\right)}\right)\|y-z\|_{C([0, a] ; X)} \\
& +C_{0} a^{2} \mathfrak{M}_{1} \mathfrak{M}_{2}\|y-z\|_{C([0, a] ; X)}+C_{0}^{2} a^{2} \mathfrak{M}_{1} \mathfrak{M}_{2} \sum_{k=1}^{n}\left(L_{I_{k}}+a L_{J_{k}}\right)\|y-z\|_{C([0, a] ; X)} \\
& +C_{0} \sum_{k=1}^{n}\left(L_{I_{k}}+a L_{J_{k}}\right)\|y-z\|_{C([0, a] ; X)} \\
\leq & C_{0}\left[2 a^{2}\left(1+C_{0} a b \mathfrak{M}_{1} \mathfrak{M}_{2}\right) L_{F}\left(1+\mathcal{R}[\sigma]_{C_{L i p}\left([0, b] \times \mathscr{B} ; \mathbb{R}^{+}\right)}\right)\right. \\
& \left.+a^{2} \mathfrak{M}_{1} \mathfrak{M}_{2}+\left(1+C_{0} a \mathfrak{M}_{1} \mathfrak{M}_{2}\right) \sum_{k=1}^{n}\left(L_{I_{k}}+a L_{J_{k}}\right)\right] d(y, z) .
\end{aligned}
$$

$$
\begin{aligned}
\left\|(\mathcal{G} y)^{\prime}(t)-(\mathcal{G} z)^{\prime}(t)\right\| \leq & \int_{0}^{t} C_{0} L_{F}\left(\left\|y_{\sigma\left(s, y_{s}\right)}-z_{\sigma\left(s, v_{s}\right)}\right\| \mathscr{B}+\left\|y_{\sigma\left(s, y_{s}\right)}^{\prime}-z_{\sigma\left(s, z_{s}\right)}^{\prime}\right\| \mathscr{B}\right) d s \\
& +\int_{0}^{t} C_{0} \mathfrak{M}_{1} \mathfrak{M}_{2}\left[\|y(b)-z(b)\|+\int_{0}^{b} C_{0} a L_{F}\left(\left\|y_{\sigma\left(s, y_{s}\right)}-z_{\sigma\left(s, z_{s}\right)}\right\| \mathscr{B}+\left\|y_{\sigma\left(s, y_{s}\right)}^{\prime}-z_{\sigma\left(s, z_{s}\right)}^{\prime}\right\|_{\mathscr{B}}\right) d s\right. \\
& \left.+C_{0} \sum_{k=1}^{n}\left\|I_{k}\left(y\left(t_{k}\right)\right)-I_{k}\left(z\left(t_{k}\right)\right)\right\|+C_{0} a \sum_{k=1}^{n}\left\|J_{k}\left(y\left(t_{k}\right)\right)-J_{k}\left(z\left(t_{k}\right)\right)\right\|\right](\xi) d \xi \\
& +\mathscr{A} C_{0} a \sum_{k=1}^{n}\left\|I_{k}\left(y\left(t_{k}\right)\right)-I_{k}\left(z\left(t_{k}\right)\right)\right\|+C_{0} \sum_{k=1}^{n}\left\|J_{k}\left(y\left(t_{k}\right)\right)-J_{k}\left(z\left(t_{k}\right)\right)\right\| \\
\leq & C_{0}\left[2 a\left(1+C_{0} a b \mathfrak{M}_{1} \mathfrak{M}_{2}\right) L_{F}\left(1+\mathcal{R}[\sigma]_{C_{L i p}\left([0, b] \times \mathscr{B} ; \mathbb{R}^{+}\right)}\right)+a \mathfrak{M}_{1} \mathfrak{M}_{2}\right. \\
& \left.+C_{0} a \mathfrak{M}_{1} \mathfrak{M}_{2} \sum_{k=1}^{n}\left(L_{I_{k}}+a L_{J_{k}}\right)+\sum_{k=1}^{n}\left(\mathscr{A} a L_{I_{k}}+L_{J_{k}}\right)\right] d(y, z) .
\end{aligned}
$$

From (20) and (21), we get

$$
d(\mathcal{G} y, \mathcal{G} z) \leq \Lambda d(y, z) .
$$

This shows the contraction map $\mathcal{G}$, which implies the map $\mathcal{G}$ is a condensing operator on the interval $[0, a]$ such that $y \in C_{L i p}([-\gamma, a] ; X)$. From Lemma $2, \mathcal{G}$ has a fixed point in $X$. Thus, any fixed point of $\mathcal{G}$ is a mild solution of (1)-(4). Hence the system (1)-(4) is controllable on $[-\gamma, a]$. 


\section{Wellposedness}

The metric space $\left(\mathcal{M},\|\cdot\|_{\mathcal{M}}\right)$ is continuous and is defined on $C([-\gamma, 0] ; X)$. Now, suppose that $\omega^{\prime}(0)$ exists for every $\omega \in \mathcal{M}$.

Definition 3. If for every pair of open and bounded convex subset $S$ of $\mathcal{M}$, there is any $a_{S}>0$, in order to allow all $\omega \in S$ and there is exactly one mild solution $y^{\omega} \in C^{1}\left(\left[-\gamma, a_{S}\right] ; X\right)$ of (1) with the conditions $y_{0}=\omega, y^{\prime}\left(0^{+}\right)=\omega^{\prime}(0)$ and $\left\|y^{\omega}-y^{\phi}\right\|_{C^{1}\left(\left[0, a_{S}\right] ; X\right)} \rightarrow 0$ as $\|\omega-\phi\|_{\mathcal{M}} \rightarrow 0$, then the problem (1) is called wellposed on the neighborhood of $\mathcal{M}$.

The remaining results, $\mathcal{M}_{\mathscr{B}}$ denotes that

$\mathcal{M}_{\mathscr{B}}=\left\{\omega \in C_{\text {Lip }}^{1}([-\gamma, 0] ; X): \omega(0) \in \mathcal{E},\left\{C(\cdot) \omega(0), \mathscr{A} S(\cdot) \omega(0), C(\cdot) \omega^{\prime}(0)\right\} \subset C_{\text {Lip }}([0, b] ; X)\right\}$,

provided by taking $\mathbb{D}(\omega, \phi)=\|\omega-\phi\|_{C_{L i p}^{1}([-\gamma, 0] ; X)}+\|C(\cdot)(\omega(0)-\phi(0))\|_{C_{L i p}([0, b] ; X)}$ $+\|\mathscr{A} S(\cdot)(\omega(0)-\phi(0))\|_{C_{L i p}([0, b] ; X)}+\left\|C(\cdot)\left(\omega^{\prime}(0)-\phi^{\prime}(0)\right)\right\|_{\left.C_{L i p}(0, b] ; X\right)}$.

Further, we show the consequence of wellposedness and it is proved from Theorem 1. Now, we introduce the following hypothesis to demonstrate our next result of condition $\left(H_{\sigma, \varphi}\right)$.

$\left(H_{\sigma}\right) \sigma \in C_{L i p}\left([0, b] \times \mathscr{B} ; \mathbb{R}^{+}\right), \sigma(0, \omega)=0$ for every $\omega \in \mathscr{B}$ and for every open set $\mathcal{U} \subset \mathscr{B}$ is bounded and there is a $0<a_{\mathcal{U}}^{*} \leq b$ provides $0 \leq \sigma(t, \omega) \leq t$, for every $(t, \omega) \in\left[0, a_{\mathcal{U}}^{*}\right] \times \mathcal{U}$.

Theorem 2. Suppose the condition $H_{\sigma}$ holds and $\mathscr{F} \in C_{L i p}([0, b] \times \mathscr{B} \times \mathscr{B} ; X)$. Then the problem (1)-(4) is locally wellposed on $\mathcal{M}_{\mathscr{B}}$.

Proof. Let $S \subset \mathcal{M}_{X}$ be open, bounded, convex sets, $S_{1}=S+B_{1}(0, \mathscr{B}) \subset \mathscr{B}$ and the number $a_{S_{1}}^{*}$ is taken from condition $H_{\sigma}$. Let $\mathcal{R}_{S}>0$ be sufficiently large number such that $\mathcal{R}_{S}>\Theta=\sup _{\omega \in S}\left([\omega]_{C_{L i p}^{1}([-\gamma, 0] ; X)}+\mathbb{D}(\omega, 0)+C_{0}\left\|\omega^{\prime}(0)\right\|+2 C_{0}\left(2 L_{F}+\sup _{\tau \in[0, b]}\right) \|\right.$ $\mathscr{F}\left(\tau, \omega, \omega^{\prime}\right) \|+C_{0} \sum_{k=1}^{n}\left(\Psi_{k}\left(a_{S_{1}}^{*}\right)\right)$. Now, we select that $0<a<\min \left\{b, a_{S_{1}}^{*}, 1\right\}$ such that $\mathcal{R}_{S} a_{S} \leq \min \left\{b, a_{S_{1}}^{*}, 1\right\}$ and

$$
\begin{gathered}
\mathrm{Y}=2 C_{0} a_{S}\left(1+a_{S}\right)\left(1+C_{0} a_{S} b_{S} \mathfrak{M}_{1} \mathfrak{M}_{2}\right) L_{F}\left(1+\mathcal{R}_{S}[\sigma]_{C_{L i p}\left([0, b] \times \mathscr{B} ; \mathbb{R}^{+}\right)}\right) \\
\Theta+a_{S} C_{0}\left(1+C_{0} a_{S} b_{S} \mathfrak{M}_{1} \mathfrak{M}_{2}\right) L_{F}\left(1+2 \mathcal{R}_{S}[\sigma]_{C_{L i p}\left([0, b] \times \mathscr{B} ; \mathbb{R}^{+}\right)}\left(1+\mathcal{R}_{S}\right)\right) \leq \mathcal{R}_{S} .
\end{gathered}
$$

Let $\omega \in S$ and let $\mathcal{Y}_{\omega}\left(a, \mathcal{R}_{S}\right)$ be the space defined by

$$
\begin{gathered}
\mathcal{Y}_{\omega}\left(a_{S}, \mathcal{R}_{S}\right)=\left\{y \in C^{1}\left(\left[-\gamma, a_{S}\right] ; X\right): y_{0}=\omega, \max \left\{[y]_{\mathcal{L}_{L i p}\left(\left[-\gamma, a_{S}\right] ; X\right),}\left[y^{\prime}\right]_{C_{L i p}\left(\left[-\gamma, a_{S}\right] ; X\right)}\right\} \leq \mathcal{R}_{S}\right\}, \\
\text { awarded with the metric } d(y, z)=\|y-z\|_{C\left(\left[0, a_{S}\right] ; X\right)} \text { and if } \mathcal{G}_{\omega}: \mathcal{Y}_{\omega}\left(a_{S}, \mathcal{R}_{S}\right) \\
\rightarrow C\left(\left[-\gamma, a_{S}\right] ; X\right) \text { is given by }\left(\mathcal{G}_{\omega} y\right)_{0}=\omega \text { and } \\
\mathcal{G}_{\omega} y(t)=C(t) \omega(0)+S(t) \omega^{\prime}(0)+\int_{0}^{t} S(t-s) \mathscr{F}\left(s, y_{\sigma\left(s, y_{s}\right)}, y_{\sigma\left(s, y_{s}\right)}^{\prime}\right) d s+\int_{0}^{t} S(t-\xi) B W^{-1}\{y(b)-C(b) \omega(0) \\
\left.-S(b) \omega^{\prime}(0)-\int_{0}^{b} S(b-s) \mathscr{F}\left(s, y_{\sigma\left(s, y_{s}\right)}, y_{\sigma\left(s, y_{s}\right)}^{\prime}\right) d s-\sum_{k=1}^{n} C\left(b-t_{k}\right) I_{k}\left(y_{t_{k}}\right)-\sum_{k=1}^{n} S\left(b-t_{k}\right) J_{k}\left(y_{t_{k}}\right)\right\}(\xi) d \xi \\
+\sum_{0<t_{k}<t} C\left(t-t_{k}\right) I_{k}\left(y_{t_{k}}\right)+\sum_{0<t_{k}<t} S\left(t-t_{k}\right) J_{k}\left(y_{t_{k}}\right), \text { for } t \in\left[0, a_{S}\right] .
\end{gathered}
$$

Let $y \in \mathcal{Y}_{\omega}\left(a_{S}, \mathcal{R}_{S}\right)$. For $t \in\left[0, a_{S}\right],\left\|y_{t}-\omega\right\|_{\mathscr{B}} \leq \mathcal{R}_{S} a_{S} \leq a_{S_{1}}^{*}$, gives that $0 \leq$ $\sigma\left(t, y_{t}\right) \leq t \leq a_{S}$ and that the functions $y_{\sigma\left(\cdot, y_{(\cdot)}\right)}, y_{\sigma(\cdot, y(\cdot)}^{\prime}, \mathscr{F}\left(\cdot, y_{\sigma(\cdot, y(\cdot))}, y_{\sigma(\cdot, y(\cdot))}^{\prime}\right)$ and $\mathcal{G}_{\omega} y(\cdot)$ are well defined. From Theorem 1, we get

$$
\sup _{s \in\left[0, a_{S}\right]}\left\|\mathscr{F}\left(s, y_{\sigma\left(s, y_{s}\right)}, y_{\sigma\left(s, y_{s}\right)}^{\prime}\right)\right\| \leq 2 L_{F}+\sup _{\tau \in[0, a]}\left\|\mathscr{F}\left(\tau, \omega, \omega^{\prime}\right)\right\| .
$$


By using the proof of Theorem 1 and the Equations (25) and (26), we have

$$
\begin{aligned}
{\left[\mathcal{G}_{\omega} y\right]_{C_{L i p}\left(\left[0, a_{S}\right] ; X\right) \leq} \leq } & \sup _{\phi \in S}\left([C(\cdot) \phi(0)]_{C_{L i p}\left(\left[0, a_{S}\right] ; X\right)}\right)+C_{0} a\left\|\phi^{\prime}(0)\right\|+C_{0} a^{2}\left(2 L_{F}+\sup _{\tau \in\left[0, b_{S}\right]}\left\|\mathscr{F}\left(\tau, \phi, \phi^{\prime}\right)\right\|\right) \\
& +C_{0} a^{2} \mathfrak{M}_{1} \mathfrak{M}_{2} K_{b}+C_{0} \sum_{k=1}^{n}\left(\Phi_{k}\left(a_{S_{1}}^{*}\right)+a_{S} \Psi_{k}\left(a_{S_{1}}^{*}\right)\right)
\end{aligned}
$$

$$
\begin{aligned}
& {\left[\left(\mathcal{G}_{\omega} y\right)^{\prime}\right]_{C_{L i p}\left(\left[0, a_{S}\right] ; X\right)} \leq \sup _{\phi \in S}\left([\mathscr{A} S(\cdot)]_{C_{L i p}\left(\left[0, a_{S}\right] ; X\right)}+\left[C(\cdot) \phi^{\prime}(0)\right]_{C_{L i p}\left(\left[0, a_{S}\right] ; X\right)}\right)+C_{0} a_{S}\left(2 L_{F}+\sup _{\tau \in\left[0, b_{S}\right]}\left\|\mathscr{F}\left(\tau, \phi, \phi^{\prime}\right)\right\|\right)} \\
& +C_{0} a_{S} \mathfrak{M}_{1} \mathfrak{M}_{2} K_{b}+C_{0} \sum_{k=1}^{n}\left(\mathscr{A} a_{S} \Phi_{k}\left(a_{S_{1}}^{*}\right)+a_{S} \Psi_{k}\left(a_{S_{1}}^{*}\right)\right), \\
& \text { and } \\
& \left\|\mathcal{G}_{\omega} y-\mathcal{G}_{\omega} z\right\|_{C\left(\left[0, a_{S}\right] ; X\right)} \leq\left[2 C_{0} a_{S}^{2} L_{F}\left(1+C_{0} a_{S} b_{S} \mathfrak{M}_{1} \mathfrak{M}_{2}\right)\left(1+\mathcal{R}_{S}[\sigma]_{C_{L i p}\left(\left[0, b_{S}\right] \times \mathscr{B} ; \mathbb{R}^{+}\right)}\right)\right. \\
& \left.+C_{0} a_{S}^{2} \mathfrak{M}_{1} \mathfrak{M}_{2}+\left(1+C_{0} a_{S} \mathfrak{M}_{1} \mathfrak{M}_{2}\right) \sum_{k=1}^{n}\left(L_{I_{k}}+a_{S} L_{J_{k}}\right)\right] d(y, z), \\
& \left\|\left(\mathcal{G}_{\omega} y\right)^{\prime}-\left(\mathcal{G}_{\omega} z\right)^{\prime}\right\|_{C\left(\left[0, a_{S}\right] ; X\right)} \leq C_{0}\left[2 a_{S} L_{F}\left(1+C_{0} a_{S} b_{S} \mathfrak{M}_{1} \mathfrak{M}_{2}\right)\left(1+\mathcal{R}_{S}[\sigma]_{C_{L i p}\left([0, b] \times \mathscr{B} ; \mathbb{R}^{+}\right)}\right)\right. \\
& \left.+C_{0} a_{S} \mathfrak{M}_{1} \mathfrak{M}_{2}+C_{0} a_{S} \mathfrak{M}_{1} \mathfrak{M}_{2} \sum_{k=1}^{n}\left(L_{I_{k}}+a_{S} L_{J_{k}}\right)+\sum_{k=1}^{n}\left(\mathscr{A} a_{S} L_{I_{k}}+L_{J_{k}}\right)\right] d(y, z)
\end{aligned}
$$

By the Equations (23), (27) and (28), which shows that $\left[\left(\mathcal{G}_{\omega} y\right)\right]_{C_{L i p}\left(\left[-\gamma, a_{S}\right] ; X\right)} \leq \mathcal{R}_{S}$, $\left[\left(\mathcal{G}_{\omega} y\right)^{\prime}\right]_{C_{L i p}\left(\left[-\gamma, a_{S}\right] ; X\right)} \leq \mathcal{R}_{S}, \mathcal{G}_{\omega}$ is a $\mathcal{Y}_{\omega}\left(a_{S}, \mathcal{R}_{S}\right)$-valued function. From (22), (29) and (30), we have

$$
d\left(\mathcal{G}_{\omega} y, \mathcal{G}_{\omega} z\right) \leq \mathrm{Y} d(y, z) .
$$

Thus, there exists a mild solution $y^{\omega} \in C_{L i p}^{1}([-\gamma, a] ; X)$ of $(1)-(4)$ on $\left[0, a_{S}\right]$ with the states $\omega$ and $\omega^{\prime}(0)$.

Contrarily, we get the following result from Lemma 1 with $\phi, \omega \in S$. For $t \in\left[0, a_{S}\right]$, we have

$$
\begin{aligned}
\| y^{\omega}(t) & -y^{\phi}(t) \| \\
\leq \quad & \|C(t)(\phi(0)-\omega(0))\|+\left\|S(t)\left(\phi^{\prime}(0)-\omega^{\prime}(0)\right)\right\| \\
& +\int_{0}^{t} C_{0} a_{S} L_{F}\left(\left\|y_{\sigma\left(s, y_{s}^{\omega}\right)}^{\omega}-y_{\sigma\left(s, y_{s}^{\phi}\right)}^{\phi}\right\|_{\mathscr{B}}+\left\|\left(y^{\omega}\right)_{\sigma\left(s, y_{s}^{\omega}\right)}^{\prime}-\left(y^{\phi}\right)_{\sigma\left(s, y_{s}^{\phi}\right)}^{\prime}\right\|_{\mathscr{B}}\right) d s \\
& +\int_{0}^{t} C_{0} a_{S} \mathfrak{M}_{1} \mathfrak{M}_{2}\left[\left\|y^{\omega}(b)-y^{\phi}(b)\right\|_{\mathscr{B}}+\|C(t)(\phi(0)-\omega(0))\|+\left\|S(t)\left(\phi^{\prime}(0)-\omega^{\prime}(0)\right)\right\|\right. \\
& +\int_{0}^{b} C_{0} a_{S} L_{F}\left(\left\|y_{\sigma\left(s, y_{s}^{\omega}\right)}^{\omega}-y_{\sigma\left(s, y_{s}^{\phi}\right)}^{\phi}\right\|_{\mathscr{B}}+\left\|\left(y^{\omega}\right)_{\sigma\left(s, y_{s}^{\omega}\right)}^{\prime}-\left(y^{\phi}\right)_{\sigma\left(s, y_{s}^{\phi}\right)}^{\prime}\right\|_{\mathscr{B}}\right) d s \\
& \left.+C_{0} \sum_{k=1}^{n}\left\|I_{k}\left(y_{t_{k}}^{\omega}\right)-I_{k}\left(y_{t_{k}}^{\phi}\right)\right\|_{\mathscr{B}}+C_{0} a_{S} \sum_{k=1}^{n}\left\|J_{k}\left(y_{t_{k}}^{\omega}\right)-J_{k}\left(y_{t_{k}}^{\phi}\right)\right\|_{\mathscr{B}}\right](\xi) d \xi \\
& +C_{0} \sum_{k=1}^{n}\left\|I_{k}\left(y_{t_{k}}^{\omega}\right)-I_{k}\left(y_{t_{k}}^{\phi}\right)\right\|_{\mathscr{B}}+C_{0} a_{S} \sum_{k=1}^{n}\left\|J_{k}\left(y_{t_{k}}^{\omega}\right)-J_{k}\left(y_{t_{k}}^{\phi}\right)\right\|_{\mathscr{B}} \\
\leq \quad & \left(1+C_{0} a_{S}^{2} \mathfrak{M}_{1} \mathfrak{M}_{2}\right) \mathbb{D}(\phi, \omega)+\left[2 C_{0} a_{S}^{2}\left(1+C_{0} a_{S}^{2} b_{S} \mathfrak{M}_{1} \mathfrak{M}_{2}\right) L_{F}\left(1+\mathcal{R}_{S}[\sigma]_{C_{L i p}\left([0, b] \times \mathscr{B} ; \mathbb{R}^{+}\right)}\right)\right. \\
& \left.+C_{0} a_{S}^{2} \mathfrak{M}_{1} \mathfrak{M}_{2}+C_{0}\left(1+C_{0} a_{S}^{2} \mathfrak{M}_{1} \mathfrak{M}_{2}\right) \sum_{k=1}^{n}\left[L_{I_{k}}+a_{S} L_{J_{k}}\right]\right]\left\|y^{\omega}-y^{\phi}\right\|_{C^{1}\left(\left[-\gamma, a_{S}\right] ; X\right)},
\end{aligned}
$$




$$
\begin{aligned}
\|\left(y^{\omega}\right)^{\prime} & (t)-\left(y^{\phi}\right)^{\prime}(t) \| \\
\leq & \|\mathscr{A} S(\cdot)(\phi(0)-\omega(0))\|+\left\|C(\cdot)\left(\phi^{\prime}(0)-\omega^{\prime}(0)\right)\right\| \\
& +\int_{0}^{t} C_{0} L_{F}\left(\left\|y_{\sigma\left(s, y_{s}^{\omega}\right)}^{\omega}-y_{\sigma\left(s, y_{s}^{\phi}\right)}^{\phi}\right\|_{\mathscr{B}}+\left\|\left(y^{\omega}\right)_{\sigma\left(s, y_{s}^{\omega}\right)}^{\prime}-\left(y^{\phi}\right)_{\sigma\left(s, y_{s}^{\phi}\right)}^{\prime}\right\|_{\mathscr{B}}\right) d s \\
& +\int_{0}^{t} C_{0} \mathfrak{M}_{1} \mathfrak{M}_{2}\left[\left\|y^{\omega}(b)-y^{\phi}(b)\right\|_{\mathscr{B}}+C_{0}\left(\|\phi-\omega\|_{\mathscr{B}}+a_{S}\left\|\phi^{\prime}-\omega^{\prime}\right\|_{\mathscr{B}}\right)\right. \\
& +\int_{0}^{b} C_{0} a_{S} L_{F}\left(\left\|y_{\sigma\left(s, y_{s}^{\omega}\right)}^{\omega}-y_{\sigma\left(s, y_{s}^{\phi}\right)}^{\phi}\right\|_{\mathscr{B}}+\left\|\left(y^{\omega}\right)_{\sigma\left(s, y_{s}^{\omega}\right)}^{\prime}-\left(y^{\phi}\right)_{\sigma\left(s, y_{s}^{\phi}\right)}^{\prime}\right\|_{\mathscr{B}}\right) d s \\
& \left.+C_{0} \sum_{k=1}^{n}\left\|I_{k}\left(y_{t_{k}}^{\omega}\right)-I_{k}\left(y_{t_{k}}^{\phi}\right)\right\|_{\mathscr{B}}+C_{0} a_{S} \sum_{k=1}^{n}\left\|J_{k}\left(y_{t_{k}}^{\omega}\right)-J_{k}\left(y_{t_{k}}^{\phi}\right)\right\|_{\mathscr{B}}\right](\xi) d \xi \\
& +C_{0} \mathscr{A} a_{S} \sum_{k=1}^{n}\left\|I_{k}\left(y_{t_{k}}^{\omega}\right)-I_{k}\left(y_{t_{k}}^{\phi}\right)\right\|_{\mathscr{B}}+C_{0} \sum_{k=1}^{n}\left\|J_{k}\left(y_{t_{k}}^{\omega}\right)-J_{k}\left(y_{t_{k}}^{\phi}\right)\right\|_{\mathscr{B}} \\
\leq \quad & \left(1+C_{0}^{2} a_{S} \mathfrak{M}_{1} \mathfrak{M}_{2}\right) \mathbb{D}(\phi, \omega)+\left[2 C_{0} a_{S}\left(1+C_{0} a_{S} b_{S} \mathfrak{M}_{1} \mathfrak{M}_{2}\right) L_{F}\left(1+\mathcal{R}_{S}[\sigma]_{C_{L i p}\left([0, b] \times \mathscr{B} ; \mathbb{R}^{+}\right)}\right)\right. \\
& \left.+C_{0} a_{S} \mathfrak{M}_{1} \mathfrak{M}_{2}+C_{0}^{2} a_{S} \mathfrak{M}_{1} \mathfrak{M}_{2} \sum_{k=1}^{n}\left[L_{I_{k}}+a_{S} L_{J_{k}}\right]+C_{0} \sum_{k=1}^{n}\left[\mathscr{A}_{S} a_{I_{k}}+L_{J_{k}}\right]\right]\left\|y^{\omega}-y^{\phi}\right\|_{C^{1}\left(\left[-\gamma, a_{S}\right] ; X\right)},
\end{aligned}
$$

From the Equations (31) and (32), we get

$$
\begin{aligned}
& \left\|y^{\omega}(t)-y^{\phi}(t)\right\|_{C\left(\left[0, a_{S}\right] ; X\right)}+\left\|\left(y^{\omega}\right)^{\prime}(t)-\left(y^{\phi}\right)^{\prime}(t)\right\|_{C\left(\left[0, a_{S}\right] ; X\right)} \\
& \leq \quad\left(2+C_{0} a_{S}\left(C_{0}+a_{S}\right) \mathfrak{M}_{1} \mathfrak{M}_{2}\right) \mathbb{D}(\phi, \omega)+\left[2 C _ { 0 } a _ { S } ( 1 + a _ { S } ) ( 1 + C _ { 0 } a _ { S } b _ { S } \mathfrak { M } _ { 1 } \mathfrak { M } _ { 2 } ) L _ { F } \left(1+\mathcal{R}_{S}[\sigma]_{C_{L i p}}\left([0, b] \times \mathscr{B} ; \mathbb{R}^{+}\right)\right.\right. \\
& \quad+C_{0} a_{S}\left(1+a_{S}\right) \mathfrak{M}_{1} \mathfrak{M}_{2}+C_{0}\left(1+C_{0} a_{S} \mathfrak{M}_{1} \mathfrak{M}_{2}+C_{0} a_{S}^{2} \mathfrak{M}_{1} \mathfrak{M}_{2}\right) \sum_{k=1}^{n}\left(L_{I_{k}}+L_{J_{k}}\right) \\
& \left.\quad+C_{0} \sum_{k=1}^{n}\left(\mathscr{A} a_{S} L_{I_{k}}+L_{J_{k}}\right)\right]\left\|y^{\omega}-y^{\phi}\right\|_{C^{1}\left(\left[-\gamma, a_{S}\right] ; X\right)}
\end{aligned}
$$

from which we conclude that

$$
\left\|y^{\omega}-y^{\phi}\right\|_{C^{1}\left(\left[0, a_{S}\right] ; X\right)} \leq \frac{2+C_{0} a_{S}\left(C_{0}+a_{S}\right) \mathfrak{M}_{1} \mathfrak{M}_{2}+Y}{1-Y} \mathbb{D}(\phi, \omega),
$$

and $\left\|y^{\omega}-y^{\phi}\right\|_{C^{1}\left(\left[0, a_{S}\right] ; X\right)} \rightarrow 0$ as $\mathbb{D}(\phi, \omega) \rightarrow 0$.

\section{Examples}

Let $X=L^{2}([0, \pi])$ and define $\mathcal{D}(\mathscr{A})=\left\{x \in H^{2}(0, \pi): x(0)=x(\pi)=0\right\}$. The infinitesimal generator $\mathscr{A}: \mathcal{D}(\mathscr{A}) \rightarrow X$ of a strongly continuous cosine function $(C(t))_{t \in \mathbb{R}}$ on $X$ given by $\mathscr{A} x=x^{\prime \prime}$ and $\mathscr{A} x=-\sum_{n=1}^{\infty} n^{2}\left\langle x, y_{n}\right\rangle y_{n}$, for $x \in \mathcal{D}(\mathscr{A})$. Furthermore, $\mathscr{A}$ has a distinct eigenvalues of the form $-n^{2}, n \in \mathbb{N}$, and its associated eigen functions defined as $y_{n}(\zeta):=\sqrt{\frac{2}{\pi}} \sin (n \zeta)$. Additionally, it holds the characteristics defined below:

(i) The sequence $\left\{y_{n}: n \in \mathbb{N}\right\}$ creates an orthonormal basis of $X$.

(ii) The cosine family $C(t) x=\sum_{n=1}^{\infty} \cos (n t)\left\langle x, y_{n}\right\rangle y_{n}$ and sine family $S(t) x=\sum_{n=1}^{\infty} \frac{\sin (n t)}{n}$ $\left\langle x, y_{n}\right\rangle y_{n}$, for $x \in \mathcal{D}(\mathscr{A})$. 
Now, analyze the following problem

$$
\begin{aligned}
\frac{\partial^{2} y}{\partial t^{2}}(t, \varsigma) & =\frac{\partial^{2} y}{\partial \varsigma^{2}}(t, \varsigma)+\mu(t, \varsigma)+f\left(t, y\left(t-\xi(t, y(t)), y^{\prime}(t-\xi(t, y(t)), \varsigma),\right.\right. \\
y(t, x) & =0, x=0, \pi \\
y(\theta, \varsigma) & =\varphi(\theta, \varsigma), \theta \in[-\gamma, 0], \\
y^{\prime}\left(0^{+}\right) & =\varphi^{\prime}(0), \\
\Delta y\left(t_{k}\right)(\varsigma) & =\int_{-\infty}^{t_{k}} a_{k}\left(t_{k}-s\right) y(s, \varsigma) d s, k=1,2, \cdots, n \\
\Delta y^{\prime}\left(t_{k}\right)(\varsigma) & =\int_{-\infty}^{t_{k}} \tilde{a}_{k}\left(t_{k}-s\right) y(s, \varsigma) d s, k=1,2, \cdots, n,
\end{aligned}
$$

for all $t \in[0, b]$ and $\varsigma \in[0, \pi]$.

We have to show that the existence of a solution of the problem (33)-(37) by using any spcified starting phase to end phase in a Banach space $X$. Let $\varphi \in \mathscr{B}=C([-\gamma, 0] ; X)$, $x \in X, \sigma_{i}:[0, \infty) \rightarrow[0, \infty), i=1,2, \alpha \in C([0, b] \times X ; \mathbb{R})$ be continuous, the Lipschitz constant $L_{f}<\infty$ and it holds the condition defined below:

The Equations (33)-(37) consists of (1)-(4),

(a) The functions $\mathscr{F}:[0, b] \times \mathscr{B} \times \mathscr{B} \rightarrow X, \sigma:[0, b] \times C([-\gamma, 0] ; \mathscr{B}) \rightarrow \mathbb{R}$ is defined as $\mathscr{F}(t, \omega, \phi)(\varsigma)=f(t, \omega(0, \varsigma), \phi(0, \varsigma))$ and $\sigma(s, \omega)=s-\xi(s, \omega(0))$. Clearly, $\mathscr{F}(\cdot)$ and $\sigma(\cdot)$ are Lipschitz.

(b) The operators $a_{k}, \tilde{a}_{k} \in C(\mathbb{R}, \mathbb{R})$ and $L_{I_{k}}:=\left(\int_{-\infty}^{0} \frac{a_{k}^{2}(s)}{g(s)} d s\right)^{\frac{1}{2}}, L_{J_{k}}:=\left(\int_{-\infty}^{0} \frac{\tilde{a}_{k}^{2}(s)}{g(s)} d s\right)^{\frac{1}{2}}$, $k=1,2, \cdots, n$, are finite. (b) by

We describe the following functions $I_{k}: \mathscr{B} \rightarrow X$ and $J_{k}: \mathscr{B} \rightarrow X$ from the condition

$$
\begin{aligned}
& I_{k}(\omega)(\varsigma)=\int_{-\infty}^{0} a_{k}(-s) \omega(s, \varsigma) d s, k=1,2, \cdots, n, \\
& J_{k}(\omega)(\varsigma)=\int_{-\infty}^{0} \tilde{a}_{k}(-s) \omega(s, \varsigma) d s, k=1,2, \cdots, n .
\end{aligned}
$$

(c) The operator $B: U \subset[0, b] \rightarrow X$ is bounded and linear, defined by

$$
(B u(t))(\varsigma)=\mu(t, \varsigma), \quad \varsigma \in[0, \pi] .
$$

A linear operator $W$ is given by

$$
(W u)(\varsigma)=\sum_{n=1}^{\infty} \int_{0}^{\pi} \frac{1}{n} \operatorname{sinns}\left(\mu(s, \varsigma), y_{n}\right) y_{n} d s, \quad \varsigma \in[0, \pi],
$$

and it has a bounded inverse $W^{-1} \in \mathscr{L}^{2}([0, b], U)$.

With the selection of $B, \mathscr{F}, \sigma_{i}, i=1,2, I_{k}, J_{k}$, the system (1)-(4) is the abstraction formulation of (33)-(37). Furthermore, the bounded linear operators $I_{k}, J_{k}, k=1,2, \cdots, n$ is described by

$$
\|\mathscr{F}(t, \cdot)\|_{\mathscr{L}(\mathscr{B}, X)} \leq L_{f}, \quad\left\|I_{k}\right\|_{\mathscr{L}(\mathscr{B}, X)} \leq L_{I_{k}}, \quad\left\|J_{k}\right\|_{\mathscr{L}(\mathscr{B}, X)} \leq L_{J_{k}} .
$$

By using the previous result and Theorem 1 , we get the next result. In this consequence, $y \in C([-\gamma, a] ; X)$ is called a mild solution of (33)-(37) defined in $[-\gamma, a]$ if $y(\cdot)$ is the mild solution of (1)-(4) in the interval $[-\gamma, a]$. Thus, the system (33)-(37) is controllable on $[-\gamma, a]$. 
Moreover, we investigate the following system

$$
\begin{aligned}
\frac{\partial^{2} y}{\partial t^{2}}(t, \varsigma) & =\frac{\partial^{2} y}{\partial \varsigma^{2}}(t, \varsigma)+\mu(t, \varsigma)+f\left(t, y(\xi(t, y(t))-r, \varsigma), y^{\prime}(\xi(t, y(t))-r, \varsigma)\right), \\
y(t, x) & =0, x=0, \pi \\
y(\theta, \varsigma) & =\varphi(\theta, \varsigma), \theta \in[-\gamma, 0] \\
y^{\prime}\left(0^{+}, \varsigma\right) & =\varphi^{\prime}(0, \varsigma), \\
\Delta y\left(t_{k}\right)(\varsigma) & =\int_{-\infty}^{t_{k}} a_{k}\left(t_{k}-s\right) y(s, \varsigma) d s, k=1,2, \cdots, n \\
\Delta y^{\prime}\left(t_{k}\right)(\varsigma) & =\int_{-\infty}^{t_{k}} \tilde{a}_{k}\left(t_{k}-s\right) y(s, \varsigma) d s, k=1,2, \cdots, n
\end{aligned}
$$

for all $\varsigma \in[0, \pi]$ and $t \in[0, b]$, here, $\varphi(\cdot), \xi(\cdot), f(\cdot), \mathscr{F}(\cdot)$ are defined as earlier and $\rho$ : $[0, b] \times \mathscr{B}$ by $\rho(s, \omega)=\xi(s, \omega)-r$, for $0<r \leq \gamma$. We get the following result from Theorem 2:

Proposition 1. Assume $\varphi \in C_{L i p}^{1}([-\gamma, 0] ; X)$ and $\varphi(0) \in \mathcal{E}$. Then there is an exactly one mild solution $y \in C^{1}([-\gamma, a] ; X)$ of $(38)-(42)$ on $[-\gamma, a]$ provides $\rho\left(s, y_{s}\right) \leq 0$ for every $s \in[0, a]$. Also, if for all bounded sets $S \subset X$ there exists $a a_{S}>0$ such that $s \leq \sigma(s, x)$, for every $x \in S$ and $s \in\left[0, a_{S}\right]$, then problem (33)-(37) is locally wellposed on the space $\mathcal{M}_{X}$.

At last, we briefly discuss the following problem

$$
\begin{aligned}
\frac{\partial^{2} y}{\partial t^{2}}(t, \varsigma) & =\frac{\partial^{2} y}{\partial \varsigma^{2}}(t, \varsigma)+\mu(t, \varsigma)+\int_{0}^{t} \alpha\left(t-s, y(t-\xi(t, y(t))+s, \varsigma), y^{\prime}(t-\xi(t, y(t))+s, \varsigma)\right) d s \\
y(t, x) & =0, \quad x=0, \pi \\
y(\theta, \varsigma) & =\varphi(\theta, \varsigma), \quad \theta \in[-\gamma, 0] \\
y^{\prime}\left(0^{+}, \varsigma\right) & =\varphi^{\prime}(0, \varsigma) \\
\Delta y\left(t_{k}\right)(\varsigma) & =\int_{-\infty}^{t_{k}} a_{k}\left(t_{k}-s\right) y(s, \varsigma) d s, k=1,2, \cdots, n \\
\Delta y^{\prime}\left(t_{k}\right)(\varsigma) & =\int_{-\infty}^{t_{k}} \tilde{a}_{k}\left(t_{k}-s\right) y(s, \varsigma) d s, k=1,2, \cdots, n
\end{aligned}
$$

for $(t, \zeta) \in[0, b] \times[0, \pi]$ and for all $t \in[0, b]$, where $\alpha \in C_{L i p}\left(\mathbb{R}^{3} ; R\right), \varphi(\cdot)$ and $\xi(\cdot)$ are defined earlier and $b \leq \gamma$. Using the above result, describe the function $\mathscr{F}(\cdot), \sigma(\cdot)$ as

$\mathscr{F}(t, \omega, \varphi)=\int_{0}^{t} \alpha(t-s, \omega(-s, \varsigma), \varphi(-s, \varsigma)) d s, \quad B u(t)=\mu(t, \varsigma) \quad$ and $\quad \sigma(t, \omega)=t-\xi(t, \omega(0))$.

Clearly, we show that $\mathscr{F}, \sigma$ are Lipschitz. We get the next consequence from Theorem 1.

Proposition 2. Suppose $\varphi \in C_{\text {Lip }}^{1}([-\gamma, 0] ; X), \mathscr{A} S(\cdot) \varphi(0), C(\cdot) \varphi^{\prime}(0), C(\cdot) \varphi(0) \in C_{\text {Lip }}([0, a] ;$ $X)$ and there is a positive number $0<r \leq b$ in the sense $0 \leq t-\xi(s, x)-s$ for every $(t, x) \in[0, r] \times B_{r}(\varphi(0) ; X)$ and $0<s \leq t$ gives the existence of exactly one mild solution $y \in C_{\text {Lip }}^{1}([-\gamma, a] ; X)$ of $(43)-(47)$ on $[-\gamma, a]$, for any $0<a \leq b$ and $y(\cdot)$ is a classical solution whenever $\varphi(0) \in \mathcal{D}(\mathscr{A})$. Furthermore, if for every $S \subset X$ bounded, there exists a number $a_{S}>0$ provides $s \leq \sigma(s, x)$ for each $x \in S$ and all $s \in\left[0, a_{S}\right]$ and if $I_{k}\left(y_{t_{k}}\right), J_{k}\left(y_{t_{k}}\right) \in H^{2}(0, \pi)$, for every $k=1,2, \cdots, n$, then problem (43)-(47) is locally wellposed on $\mathcal{M}_{X}$.

\section{Conclusions}

The conditions for controllability and wellposedness of an abstract second-order differential system with state-dependent delay are investigated in this paper. The use of the work is shown by demonstrating its application to dynamical systems. We came to the conclusion that the existence of controllability and wellposedness achieves the goal of a 
stated problem and its remedies. It will be more fascinating to study the equivalent system's trajectory controllability and numerical estimation. We plan to extend it to a fractional order system with state dependent indefinite delay and integral impulsive conditions in the future.

Author Contributions: All authors contributed equally in writing this article. All authors read and approved the final manuscript.

Funding: This research received no external funding.

Institutional Review Board Statement: Not applicable.

Informed Consent Statement: Not applicable.

Data Availability Statement: The data used to support the findings of this study are available from the corresponding author upon request.

Conflicts of Interest: The authors declare no conflict of interest.

\section{References}

1. Bainov, D.; Simeonov, P. Impulsive Differential Equations: Periodic Solutions and Applications; Longman Scientific \& Technical: Harlow, UK, 1993.

2. Benchohra, M.; Henderson, J.; Ntouyas, S.K. Impulsive Differential Equations and Inclusions; Hindawi Publishing Corporation: New York, NY, USA, 2006.

3. Lakshmikantham, V.; Bainov, D.D.; Simeonov, P. Theory of Impulsive Differential Equations; World Scientific: Hackensack, NJ, USA, 1989.

4. Arthi, G.; Balachandran, K. Controllability of second order impulsive functional differential equations with state dependent delay. Bull. Korea Math. Soc. 2011, 48, 1271-1290. [CrossRef]

5. Sakthivel, R.; Anandhi, E.R.; Mahmudov, N.I. Approximate controllability of second-order systems with state-dependent delay. Numer. Funct. Anal. Optim. 2008, 29, 1347-1362. [CrossRef]

6. Chang, Y.K.; Anguraj, A.; Karthikeyan, K. Existence for impulsive neutral integrodifferential inclusions with nonlocal initial conditions via fractional operators. Nonlinear Anal. Theory Methods Appl. 2009, 71, 4377-4386. [CrossRef]

7. Rogovchenko, Y. Nonlinear impulse evolution systems and applications to population models. J. Math. Anal. Appl. 1997, 207, 300-315. [CrossRef]

8. Li, M.; Huang, M. Approximate controllability of second-order impulisve stochastic differential equations with state dependent delay. J. Appl. Anal. Comp. 2018, 8, 598-619.

9. Mallika Arjunan, M.; Nadaf, N.Y. Existence and controllability results of damped second order impulsive functional differential systems with state dependent delay. Opusc. Math. 2014, 34, 503-522. [CrossRef]

10. Vijayakumar, V.; Murugesu, R. Controllability for a class of second-order evolution differential inclusions without compactness. Appl. Anal. 2019, 98, 1367-1385. [CrossRef]

11. Vijayakumar, V.; Panda, S.K.; Nisar, K.S.; Baskonus, H.M. Results on approximate controllability results for second-order Sobolevtype impulsive neutral differential evolution inclusions with infinite delay. Numer. Meth. Part Diff. Equ. 2021, 37, 1200-1221. [CrossRef]

12. Aiello, W.; Freedman, H.; Wu, J. Analysis of a model representing stage-structured population growth with state-dependent time delay. SIAM J. Appl. Math. 1992, 52, 855-869. [CrossRef]

13. Driver, R.D. A neutral system with state-dependent delay. J. Diff. Equ. 1984, 54, 73-86. [CrossRef]

14. Hartung, F.; Turi, J. On differentiability of solutions with respect to parameters in state-dependent delay equations. J. Diff. Equ. 1997, 135, 192-237. [CrossRef]

15. Hartung, F. Differentiability of solutions with respect to parameters in neutral differential equations with state-dependent delays. J. Math. Anal. Appl. 2006, 324, 504-524. [CrossRef]

16. Hartung, F.; Krisztin, T.; Walther, H.O.; Wu, J. Functional differential equations with state-dependent delays: Theory and applications. Handb. Diff. Equ. Ordinary Diff. Equ. 2006, 3, 435-545.

17. Hernández, E.; Prokopczyk, A.; Ladeira, L. A note on partial functional differential equations with state-dependent delay. Nonlinear Anal. Real World Appl. 2006, 7, 510-519. [CrossRef]

18. Kosovalic, N.; Magpantay, F.M.G.; Chen, Y.; Wu, J. Abstract algebraic-delay differential systems and age structured population dynamics. J. Diff. Equ. 2013, 255, 593-609. [CrossRef]

19. Rezounenko, A.V. A condition on delay for differential equations with discrete state-dependent delay. J. Math. Anal. Appl. 2012, 385, 506-516. [CrossRef]

20. Rezounenko, A.V. Partial differential equations with discrete and distributed state-dependent delays. J. Math. Anal. Appl. 2007, 326, 1031-1045. [CrossRef]

21. Rezounenko, A.V.; Wu, J. A non-local PDE model for population dynamics with state-selective delay: Local theory and global attractors. J. Comput. Appl. Math. 2006, 190, 99-113. [CrossRef] 
22. Chang, Y.K.; Arjunan, M.M.; Kavitha, V. Existence results for a second order impulsive functional differential equation with state-dependent delay. Diff. Equ. Appl. 2009, 1, 325-339. [CrossRef]

23. Hernández, E.; Azevedo, K.; O’Regan, D. On second order differential equations with state-dependent delay. Appl. Anal. 2018, 97, 2610-2617. [CrossRef]

24. Krisztin, T.; Rezounenko, A. Parabolic partial differential equations with discrete state-dependent delay: Classical solutions and solution manifold. J. Diff. Equ. 2016, 260, 4454-4472. [CrossRef]

25. Hernández, E.; Pierri, M.; Wu, J. $C^{1+\alpha}$-strict solutions and wellposedness of abstract differential equations with state dependent delay. J. Diff. Equ. 2016, 261, 6856-6882. [CrossRef]

26. Kosovalic, N.; Chen, Y.; Wu, J. Algebraic-delay differential systems: $C^{0}$-extendable submanifolds and linearization. Trans. Am. Math. Soc. 2017, 369, 3387-3419. [CrossRef]

27. Lv, Y.; Yuan, R.; Pei, Y. Smoothness of semiflows for parabolic partial differential equations with state-dependent delay. J. Diff. Equ. 2016, 260, 6201-6231. [CrossRef]

28. Buga, M.; Martin, M. The escaping disaster: A problem related to state-dependent delays. Z. Angew. Math. Phys. 2004, 55, 547-574.

29. Si, J.G.; Wang, X.P. Analytic solutions of a second-order functional-differential equation with a state derivative dependent delay. Colloq. Math. 1999, 79, 273-281. [CrossRef]

30. Arthi, G.; Park, J.H.; Jung, H.Y. Existence and controllability results for second-order impulsive stochastic evolution systems with state-dependent delay. Appl. Math. Comput. 2014, 248, 328-341. [CrossRef]

31. Chueshov, I.; Rezounenko, A. Dynamics of second order in time evolution equations with state-dependent delay. Nonlinear Anal. Theory Methods Appl. 2015, 123-124, 126-149. [CrossRef]

32. Das, S.; Pandey, D.N.; Sukavanam, N. Existence of solution and approximate controllability of a second-order neutral stochastic differential equation with state dependent delay. Act. Math. Sci. 2016, 36, 1509-1523. [CrossRef]

33. Hernández, E. Existence of solutions for a second order abstract functional differential equation with state-dependent delay. Electron. J. Diff. Equ. 2007, 2007, 1-10.

34. Radhakrishnan, B.; Balachandran, K. Controllability of neutral evolution integrodifferential systems with state dependent delay. J. Optim. Theory Appl. 2012, 153, 85-97. [CrossRef]

35. Mahmudov, N.I.; Udhayakumar, R.; Vijayakumar, V. On the Approximate Controllability of Second-Order Evolution Hemivariational Inequalities. Res. Math. 2020, 75, 1-19. [CrossRef]

36. Vijayakumar, V.; Udhayakumar, R.; Dineshkumar, C. Approximate controllability of second order nonlocal neutral differential evolution inclusions. IMA J. Math. Cont. Info. 2021, 38, 192-210. [CrossRef]

37. Chalishajar, D.; Karthikeyan, K.; Tamizharasan, D. Controllability of nonlocal impulsive functional differential equations with measure of noncompactness in Banach spaces. J. Nonlinear Sci. Appl. 2021, 14, 400-413. [CrossRef]

38. Chalishajar, D.; Karthikeyan, K.; Tamizharasan, D. Controllability of nonlocal neutral impulsive differential equations with measure of noncompactness. Int. J. Math. Anal. 2021, 15, 157-165. [CrossRef]

39. Kumar, M.S.; Bazighifan, O.; Almutairi, A.; Chalishajar, D. Philos-type oscillation results for third-order differential equation with mixed neutral terms. Mathematics 2021, 9, 1021. [CrossRef]

40. Vijayakumar, V.; Henríquez, H.R. Existence of global solutions for a class of abstract second-order nonlocal cauchy problem with impulsive conditions in Banach spaces. Numer. Funct. Anal. Optim. 2018, 39, 704-736. [CrossRef]

41. Hino, Y.; Murakami, S.; Naito, T. Functional Differential Equations with Infinite Delay; Lecture Notes in Mathematics; Springer: Berlin/Heidelberg, Germany, 1991.

42. Kisyński, J. On cosine operator functions and one parameter group of operators. Stud. Math. 1972, 44, 93-105. [CrossRef]

43. Travis, C.C.; Webb, G.F. Cosine families and abstract nonlinear second order differential equations. Acta Math. Acad. Sci. Hung. 1978, 32, 75-96. [CrossRef]

44. Vasil'ev, V.V.; Piskarev, S.I. Differential equations in Banach spaces II. Theory of cosine operator functions. J. Math. Sci. 2004, 122, 3055-3174. [CrossRef] 